\title{
Development of a point machine surveillance device for safety and maintenance improvement in urban transit signalling systems
}

\author{
H. Jo ${ }^{1}$, J. Hwang ${ }^{1}$, S. Shin ${ }^{1}$, J. Baek ${ }^{1}$, Y. Kim ${ }^{1} \&$ I. Lim ${ }^{2}$ \\ ${ }^{I}$ Train Control \& Communication Research Team, \\ Signalling \& Electrical Engineering Research Department, \\ Korea Railroad Research Institute (KRRI), Gyeonggi-do, Korea \\ ${ }^{2}$ Signal Team, DaeJeon Metropolitan Express Transit Corporation \\ (DJET), Daejeon-city, Korea
}

\begin{abstract}
In the case of Korea, it is known that the present condition of occurrence in railway accidents is more frequent than that of advanced countries on the basis of the fact that the number of casualties from railway accidents reaches 3-4 times higher compared to that of advanced countries, etc. Among them, since the risk of large-scale accidents is high due to the frequent occurrence of derailment accidents according to the obstacle in switchover at track turnouts, it is important to prevent accidents from occurring caused by the obstacle in point machine by supervising it on a real-time basis. Thus, this paper developed a point machine surveillance system which can prevent large-scale accidents such as the derailment and collision at the time of defect in point machine from occurring and can enhance efficiency of maintenance, and it described concrete contents of design and manufacturing in the main text.
\end{abstract}

Keywords: point machine, switchover, urban transit, signalling system.

\section{Introduction}

The cause of obstacle in switchover at track turnouts which occupies most of the large-scale accidents such as railway derailment and collision accident is considered to be the defect in point machine, and like this, it is considered that 
the prevention against defect in point machine, which is the safety-critical system controlling train routes safely is very important (Dward [1]). The present condition of the point machine is shown in Figure 1, and among them, those being used in Korea most frequently are NS-type electric point machines (Figure 2) and they are installed in the existing lines for the national metropolitan rapid transits and Korail. NS-type electric point machine consists of the condenser-driven induction motor, lockup-type control relay, reduction gear, fraction clutch, mechanical three phase circuit controller, turnout locking device and the manual turnout device which can operate electric point machines manually.

NS-type point machine(domestic product)
\begin{tabular}{c|c|c|c|c|c|}
\hline Classification & Motor voltage & $\begin{array}{c}\text { Control, } \\
\text { display voltage }\end{array}$ & $\begin{array}{c}\text { Operating } \\
\text { current }\end{array}$ & Switchover time & $\begin{array}{c}\text { Switchover } \\
\text { force }\end{array}$ \\
\hline NS-A & AC105/220V & DC24[V] & $7.5 \mathrm{~A}$ & Below 6 seconds & $300 K G$ \\
\hline NS-AM & AC105/220 & DC24[V] & $8.5 \mathrm{~A}$ & Below 7 seconds & $400 K G$ \\
\hline
\end{tabular}

\begin{tabular}{|c|c|c|c|c|}
\hline $\begin{array}{c}\text { Movable nose type(MJ81) France } \\
\text { Motor voltage }\end{array}$ & $\begin{array}{c}\text { Display } \\
\text { voltage }\end{array}$ & Operating current & Switchover time & Switchover force \\
\hline AC220/380V(three phase) & DC48[V] & Maximum 4.8A & $\begin{array}{c}\text { Below 3 4.3 } \\
\text { seconds }\end{array}$ & 400KG \\
\hline
\end{tabular}

\begin{tabular}{|c|c|c|c|c|}
\hline Motor voltage & Load & $\begin{array}{l}\text { Operating } \\
\text { current }\end{array}$ & Switchover time & Switchover force \\
\hline $\begin{array}{l}\text { AC220(single phase) } \\
\text { AC380V(three phase) }\end{array}$ & $1000 \mathrm{kgf}$ & $\begin{array}{c}\text { Below 2.5A } \\
(220 \mathrm{~V})\end{array}$ & $\begin{array}{l}\text { Below } 3 \sim 5 \\
\text { seconds }\end{array}$ & $400 K G$ \\
\hline
\end{tabular}

Figure 1: General present condition of the point machine.
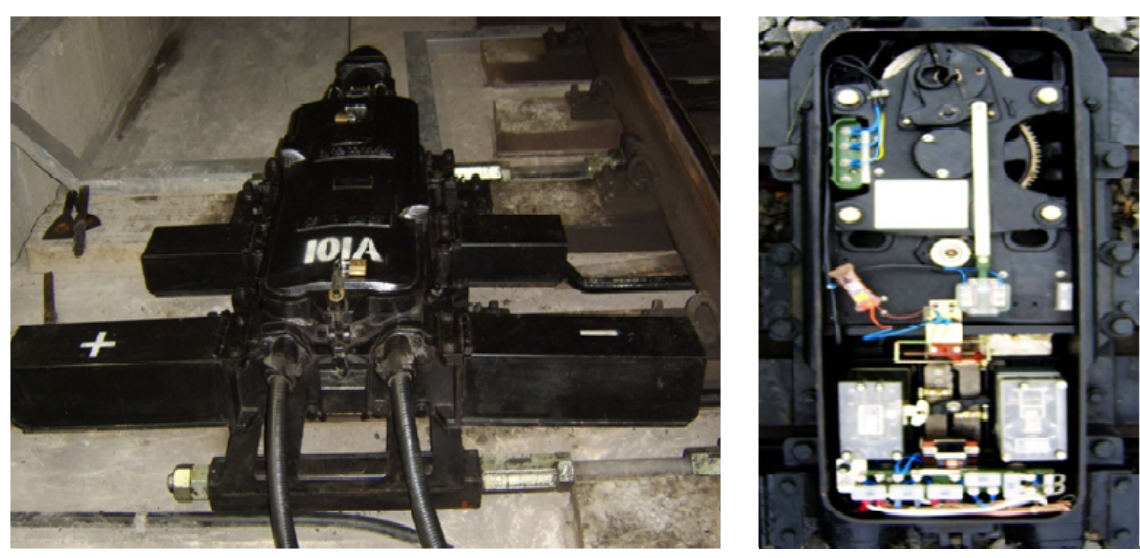

Figure 2: NS-type point machine. 
When this obstacle in point machine occurs, most of the maintenance systems so far had been normally visual and sensory checkup in the case of the mechanical system. And, in the case of the electrical system, although maintenance personnel must measure the number of failures on a real-time basis by checking and reading the control and display information of point machine according to measuring instruments, but in fact, it is difficult to conduct realtime surveillance since it is their actual situation to judge the number of failures and maintain them by measuring the voltage of six circuits (motor voltage, control voltage, display voltage, input/output voltage) simultaneously. In addition, it is the situation where improvement is necessary as [2] (Yuichiro et al. [3] and Motohashi et al. [4]) since the work is troublesome and it has the factors possible to lead to delay in recovery and safety accident also if it is measured incorrectly and misjudged.

Thus, this study developed the "real-time point machine surveillance system" which can judge immediately whether there is any abnormality existing in whole point machines in the interlocking station without any complex procedures by using the Exclusive $\mathrm{OR}(\mathrm{XOR})$ circuit and Not OR(NOR) circuit to improve these difficulties (Nelson [5]). If using the point machine surveillance system proposed, it will be possible to minimize the delay of trains and prevent safety accidents in advance since prompt and accurate recoveries will be accomplished by eliminating the troublesomeness of maintenance work when there is any obstacle occurs in the point machine. Section 2 describes functions, contents of design and results of manufacturing of the developed point machine surveillance system, and the conclusion will be made in Section 3.

\section{Design and manufacturing of the real-time point machine surveillance system}

\subsection{Features and functions of the real-time point machine surveillance system}

The developed point machine surveillance system has a feature which can judge whether there is any abnormality in voltage at each part immediately by measuring the voltage at each part of the point machine, and conduct follow-up actions against it promptly at the time of obstacle occurrence or maintenance. To this effect, to compose the surveillance system as the logic circuit to supervise the level of more than 5 units of whole point machines in one interlocking station, the control part of the proposed surveillance system was made to prevent malfunctions of other point machines when the point machine under measurement is being operated by composing it as XOR circuit (Figure 3). And, the display input, display output, control voltage and motor voltage of display part were made to express measured voltage values as digital data through interlocking with the circuit of control part by composing them as NOR circuits (Figures 4 and 5), and they were made to prevent obstacles in mixed contact due to malfunction by setting mutual interlocking between point machines. When 
organizing the function of the developed point machine surveillance system concretely, it is as follows.

- Control part of the point machine surveillance system prevents malfunctions of other point machines when the point machine under measurement is being operated by adopting the NOR which is the circuit under safety principle.

- Display part expresses measured voltage values as digital data through interlocking with the circuit of control part by being designed as the NOR circuit by linking to the control part.

- Since it is possible to measure the level automatically up to six times simultaneously, measurement of the corresponding point machine voltage shall be possible on a real-time basis by only pushing the button, and the identification shall be facilitated by adopting the digital display on the expression part.

- Although secondary induced obstacles can occur due to the transformation of elasticity in the wire connection material when tester lead rods were used continuously at the measuring points, the obstacle factor can be reduced since it is unnecessary to use the lead rod by applying the point machine surveillance system.

- Maintenance personnel can judge the number of obstacles immediately since the level will be displayed through automatic detection and measurement of the disconnection and grounding, etc. according to the state of the obstacle.

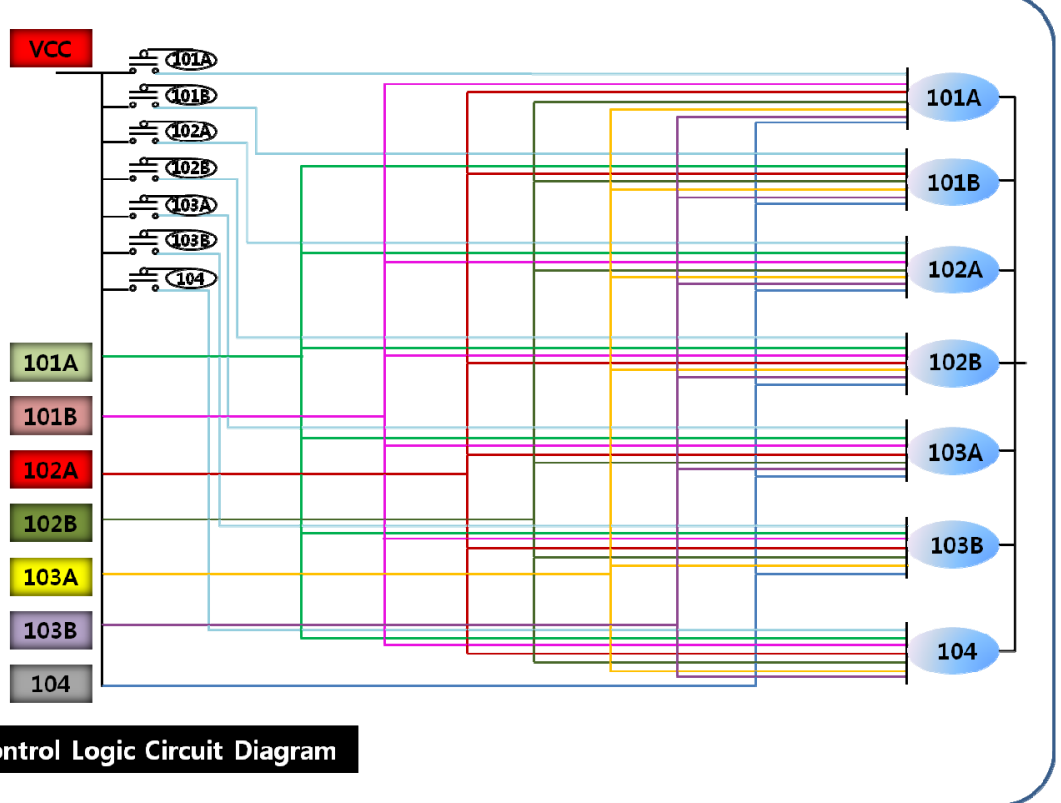

Figure 3: Logic circuit of the control part of the real-time point machine surveillance system. 


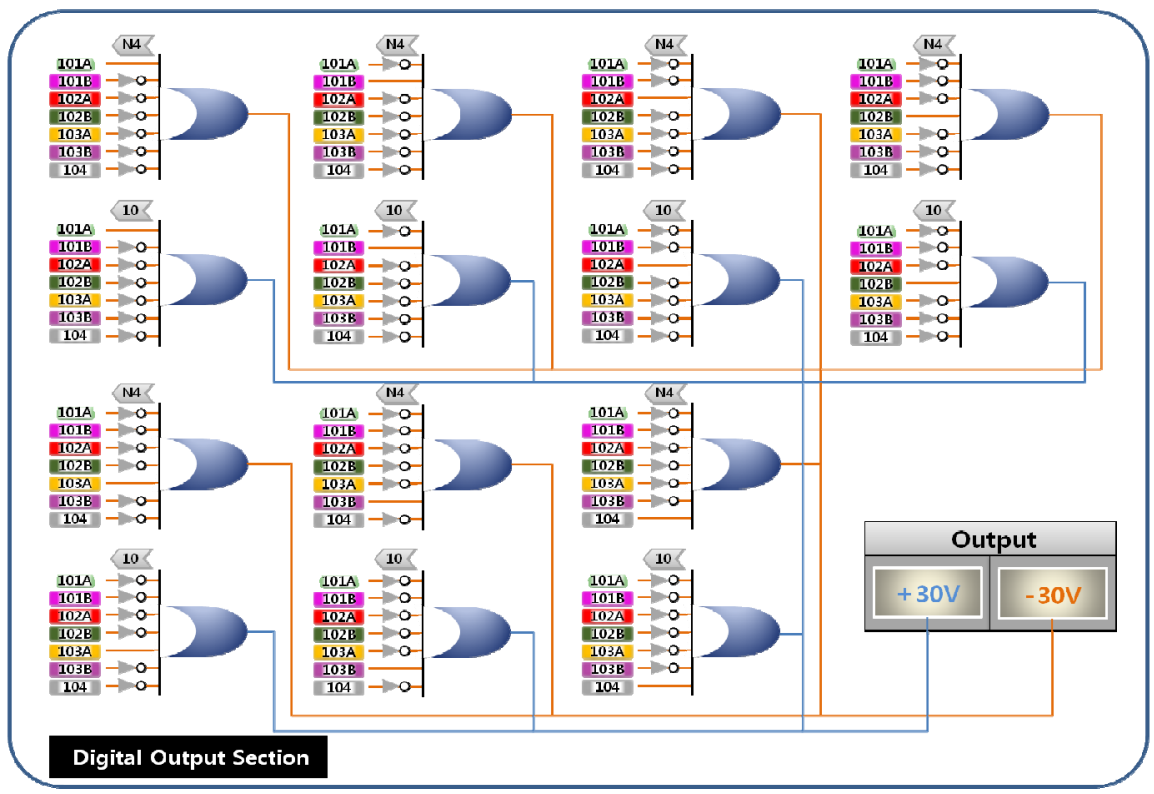

Figure 4: Logic circuit of the display part (output) of the real-time point machine surveillance system.

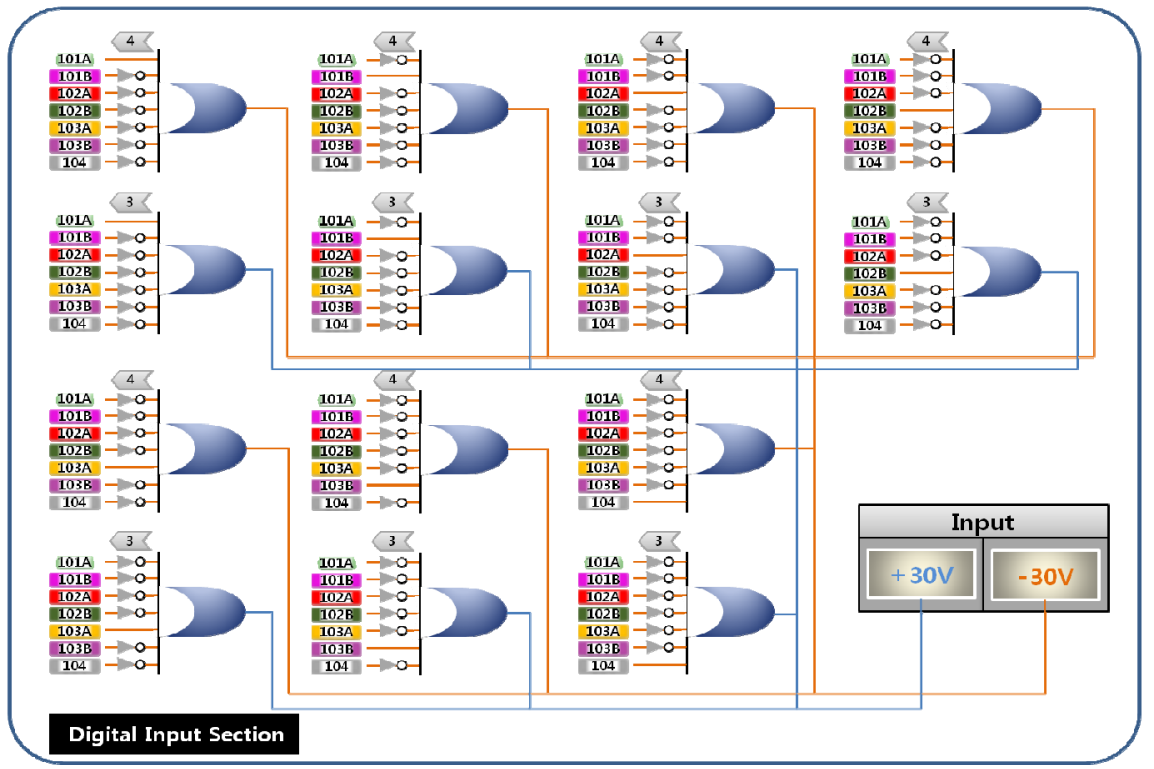

Figure 5: Logic circuit of the display part (input) of the real-time point machine surveillance system. 


\subsection{Result of the design and manufacturing of the real-time point machine surveillance system}

As mentioned in the previous section, when any obstacle occurs at the existing point machine, maintenance personnel performed works by measuring manually (Figure 6) and the delay in time for judgment was inevitable if measurement points were selected inaccurately. Due to this, there were some cases where unexpected secondary obstacles such as the damage to terminal blocks, etc. had occurred, and even the delay of trains due to the delay in recovery from the obstacle had happened frequently. Therefore, with our real-time point machine surveillance system developed in this study, difficulties in the selection of measurement points or dependence on design drawings for point machines were eliminated, and owing to the merits such as the ease of automatic measurement for six circuits only if the button was pressed and the possible automatic detection on the grounding and disconnection of circuit also, time for recovering from the obstacle was shortened effectively.
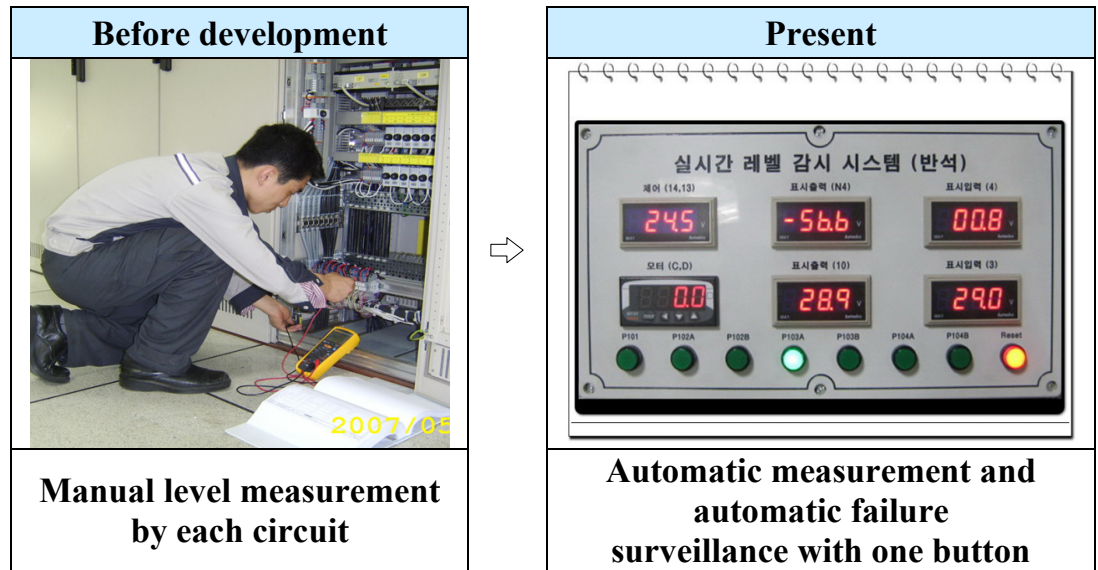

Figure 6: Comparison of the point machine surveillance system before and after development.

Specifications of design for this real-time point machine surveillance system are shown in Tables 1 and 2, and the concrete method of operation is as follows.

If looking at the operation of the real-time point machine surveillance system in detail with the example of a case in Figure 7, whether dropping contact points at internal relays of $101 \mathrm{~B}, 102 \mathrm{~A}, 102 \mathrm{~B}, 103 \mathrm{~B}, 104 \mathrm{~A}$ and $104 \mathrm{~B}$ were composed will be checked first if the measurement button for the ' 203 ' point machine is pressed. In this case, if no abnormality existed, the voltage measured at each of the digital meters will be expressed through operating of a $103 \mathrm{~A}$ relay for surveillance. Even though the button was pressed to measure other point machines, it will not be operated in accordance with its interlocking. Measurement on other point machines will be possible if only the reset button is 
Table 1: Design requirements for the point machine surveillance system.

\begin{tabular}{|c|c|c|c|c|}
\hline $\begin{array}{l}\text { Equipment } \\
\text { name }\end{array}$ & Part name & Requirements & Quantity & Remarks \\
\hline \multirow{5}{*}{$\begin{array}{l}\text { Surveillance } \\
\text { system }\end{array}$} & External box & $450 * 300 * 200$ & 1 & $\begin{array}{c}\text { Aluminum and } \\
\text { steel plates }\end{array}$ \\
\hline & Operation switch & $\begin{array}{l}16 \varphi \text { green with built- } \\
\text { in LED }\end{array}$ & 8 & $\begin{array}{c}\text { Surveillance on } \\
8 \text { point } \\
\text { machines } \\
\end{array}$ \\
\hline & Operation switch & $\begin{array}{c}16 \varphi \text { red with built-in } \\
\text { LED }\end{array}$ & 1 & \\
\hline & $\begin{array}{c}\text { Digital } \\
\text { meter(DC) }\end{array}$ & $\begin{array}{c}\text { Measurement up to } \\
199[\mathrm{~V}]\end{array}$ & 6 & $\begin{array}{l}0.2 \% \text { at full } \\
\text { scale }\end{array}$ \\
\hline & $\begin{array}{c}\text { Digital } \\
\text { meter(AC) }\end{array}$ & $\begin{array}{l}\text { Measurement up to } \\
199[\mathrm{~V}]\end{array}$ & 1 & $\begin{array}{l}0.5 \% \text { at full } \\
\text { scale }\end{array}$ \\
\hline
\end{tabular}

Table 2: $\quad$ Design requirements for a digital multi-meter.

\begin{tabular}{|c|c|c|c|}
\hline \multicolumn{2}{|c|}{ Classification } & Requirements & Remarks \\
\hline \multirow{2}{*}{ Model name } & $\begin{array}{c}\text { For DC voltage } \\
\text { measurement }\end{array}$ & M4Y -DV4 & \\
\hline & $\begin{array}{c}\text { For AC voltage } \\
\text { measurement }\end{array}$ & M4Y -AV4 & \\
\hline \multirow{2}{*}{$\begin{array}{l}\text { Measurement } \\
\text { function }\end{array}$} & DC digital meter & $\begin{array}{c}\text { Measurement of display } \\
\text { voltage at the point machine } \\
\text { Measurement of control } \\
\text { voltage at the point machine }\end{array}$ & \\
\hline & AC digital meter & $\begin{array}{c}\text { Measurement of motor } \\
\text { voltage supplied at the point } \\
\text { machine }\end{array}$ & \\
\hline \multicolumn{2}{|c|}{ Maximum display range } & Max 1999(in fixed decimals) & \\
\hline \multicolumn{2}{|c|}{ Voltage of power supply } & $100-240 \mathrm{VAC} \pm 10 \%$ & \\
\hline \multicolumn{2}{|c|}{ Power consumption } & $\mathrm{DC}: 2 \mathrm{~W}, \mathrm{AC}: 4 \mathrm{VA}$ & \\
\hline \multicolumn{2}{|c|}{ Method of display } & 7 Segment LED Display & \\
\hline \multicolumn{2}{|c|}{ Degree of display } & $\begin{array}{l}\text { DC : F.S } 0.2 \% \text { rdg } \pm 1 \text { digit } \\
\text { AC : F.S } 0.5 \% \text { rdg } \pm 1 \text { digit }\end{array}$ & F.S : Full Scale \\
\hline \multicolumn{2}{|c|}{ Sampling cycle } & $300 \mathrm{~ms}$ & \\
\hline \multicolumn{2}{|c|}{ Method of operation } & Dual slope integration mode & \\
\hline \multicolumn{2}{|c|}{ Response rate } & About 2 seconds & \\
\hline \multicolumn{2}{|c|}{ Number of display times } & 2.5 times / sec & \\
\hline \multicolumn{2}{|c|}{ Insulation resistance } & $\begin{array}{c}100[\mathrm{M} \Omega] \text { (based on } 500 \mathrm{~V} \\
\text { mega) }\end{array}$ & \\
\hline \multicolumn{2}{|c|}{ Ambient temperature } & $-10-50^{\circ} \mathrm{C}$ & \\
\hline
\end{tabular}


pressed after measuring the '203' point machine. Surveillance on the level of the point machine is accomplished in accordance with exciting contact points of relay operated by button handling, and the control of accommodating whole point machines, display 1 , display $2, \mathrm{~A}$ and $\mathrm{B}$ motor voltages can be supervised on a real-time basis. Operation of the system uses the power supply of the distribution panel with the control voltage of DC 24[V] and a display voltage of AC 110[V]. Display of the detection on failure by developing a point machine surveillance system is shown in Figure 8 in accordance with each of the following cases.

- Expresses 60[V], 30[V] if one circuit of display voltages is disconnected.

- Expresses 60[V], 60[V] if two circuits of display voltages are disconnected.

- Expresses a grounded circuit as $0[\mathrm{~V}]$ and the normal circuit as 30[V] if any display voltage is grounded.

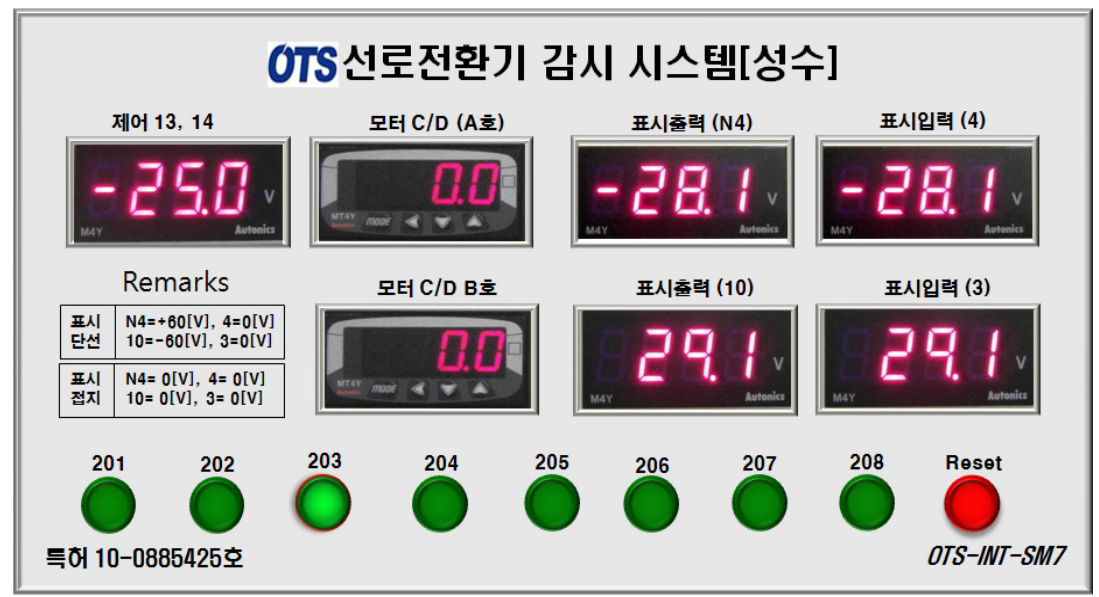

Figure 7: Operation status of the point machine surveillance system.

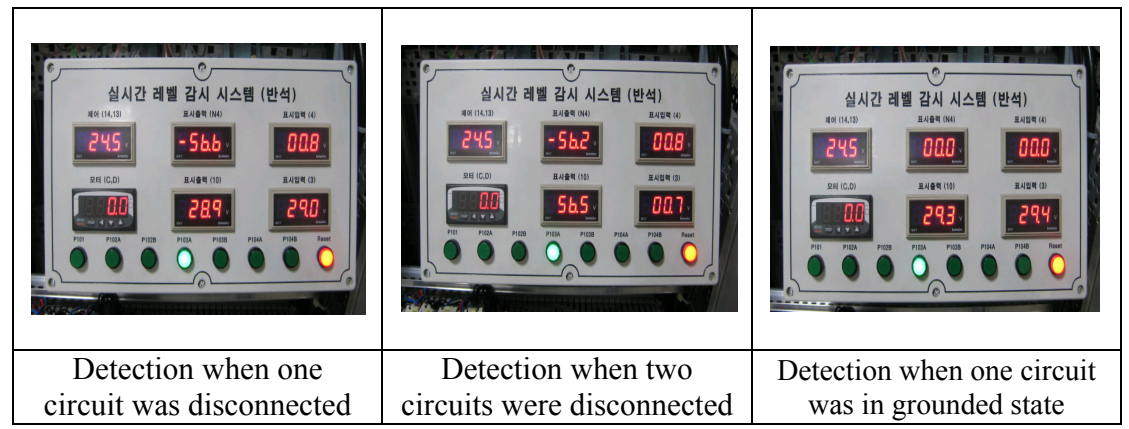

Figure 8: Display of detection on failure by the point machine surveillance system. 
However, the point machine surveillance system designed and manufactured in this study cannot store the history of measured data and to distinguish between the normal position and reverse position of the point machine, and although the surveillance is possible on a real-time basis, it is impossible to be controlled remotely and can only be controlled manually. Thus, in the future, we are planning to complement and manufacture the detection circuit by developing it with a DC voltage ranging from the minus band to the plus band so that distinction between the normal position and the reverse position is possible, and we would like to apply it to the interlocking system for display of direction in the field. And, to make surveillance on the operation status of the point machine in the unmanned interlocking station possible also, we are studying to complement it so that even real-time remote surveillance and control can be possible by transmitting its history to the remote place through RS- 485 or TCP/IP, if there is no response received for more than 16 seconds. Figure 9 below shows the plan to build our point machine surveillance system desired to be complemented.

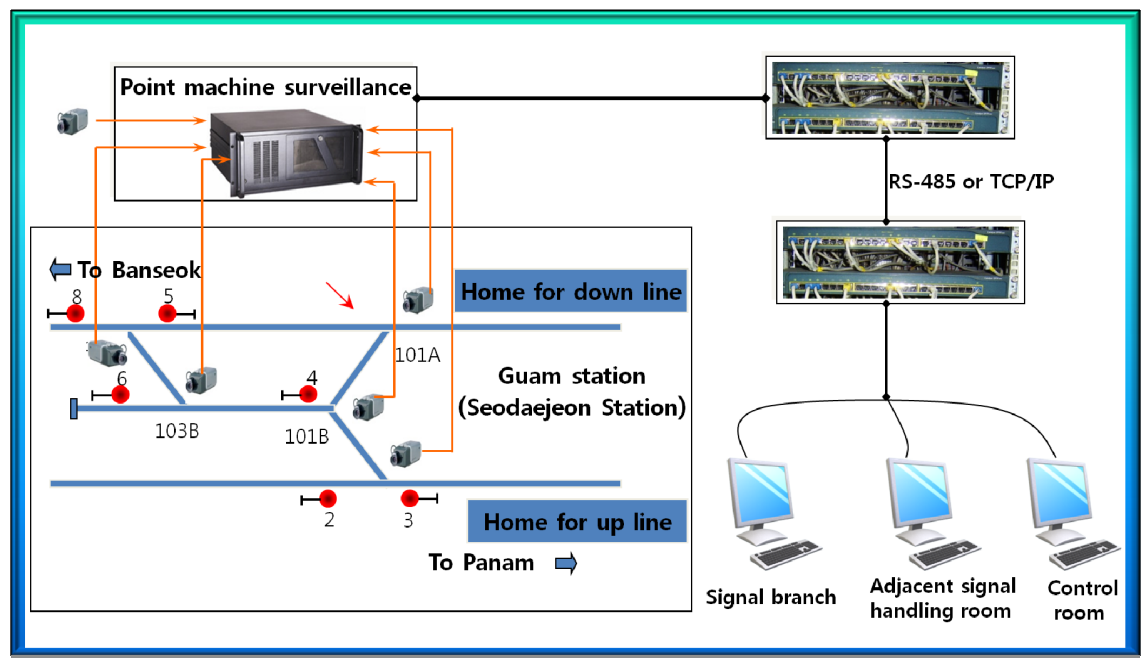

Figure 9: Configuration of the point machine surveillance system desired to be complemented.

\section{Conclusion}

This study developed a real-time point machine surveillance system to prevent the occurrence of an obstacle in track switchover that is pointed out as the main cause for occurrence of large-scale accidents such as railway derailment or collision, and to make prompt and accurate recoveries possible to be achieved by eliminating the troublesomeness of maintenance work when any obstacle occurs. Detailed functions, contents of design and the results of manufacturing the developed system are shown in the text. 
When any obstacle occurs at the NS-type electric point machine which is used a lot in Korea, maintenance workers would carry out maintenance by measuring each of the circuits manually, and the delay in time for judgment was inevitable if measurement points were selected inaccurately, and even the delay of trains occurred frequently due to the delay in recovery from the obstacle. Difficulties in the selection of measurement points or dependence on design drawings for a point machine like this were eliminated with this developed real-time point machine surveillance system, and it was made to be easy to use since six circuits will be measured automatically only if the button was pressed. Therefore, it was possible to shorten the time to recover effectively from obstacles. We are studying to make the real-time remote surveillance and control on the operation status of a point machine in unmanned interlocking stations possible at each branch office also in the future. Similarly, if we use this point machine surveillance system, it will be possible to check obstacles promptly by detecting control signals of a point machine on a real-time basis, and it will be possible to anticipate that prevention against train derailment and the safety of train operation can be secured owing to this.

\section{References}

[1] Dward Goddard, Institution of Railway Signal Engineers, Metro Railway Signalling, 2003.

[2] Signalling System Guide Book for Effective Maintenance, Daejeon Metropolitan Express Transit Corporation in Korea.

[3] Yuichiro Hori, Kazue Yasuoka, Minoru obi, Toshiyuki Kaji, Tatsuo Odaka and Koji Motohashi, "Innovation of Turnout and Switch Machine System", JR EAST Review, No. 2, pp. 31 - pp. 37.

[4] Motohashi Koji, Kaji Toshiyuki, Yasuoka Kazue, Yamaguchi Yasuhiro, Tsuchiya Masaru and Tanaka Hiroyuki; "Development of the Next Generation Electric Switch Machine," Kyosan Circular, No. 5, 2002.

[5] Nelson, Digital Logic Circuit Analysis and Design, Prentice-Hall, 1995. 